\title{
Investigation of the range of motion of the shoulder joint in subjects with rotator cuff arthropathy while performing daily activities
}

\author{
Mohammad Taghi Karimi, Sahar Khademi \\ Rehabilitation Sciences Research Center, Shiraz University of Medical Sciences, Shiraz, Iran
}

\begin{abstract}
Background: Patients who have rotator cuff arthropathy experience a limited range of motion (ROM) of the shoulder joint and experience problems in performing their daily activities; however, no evidence is available to suggest the exact ROM of the shoulder joint in this population. Therefore, this study sought to determine the degree of motion of the shoulder joint in three planes during different activities.

Methods: Five subjects with rotator cuff injuries participated in this study. The motion of the shoulder joints on both the involved and normal sides was assessed by a motion analysis system while performing forward abduction (task 1), flexion (task 2), and forward flexion (task 3). The OpenSIM software program was used to determine the ROM of the shoulder joints on both sides. The difference between the ranges of motion was determined using a two-sample t-test.

Results: The ROMs of the shoulder joint in task 1 were $93.5^{\circ} \pm 16.5^{\circ}, 72.1^{\circ} \pm 2.6^{\circ}$, and $103.9^{\circ} \pm 25.7^{\circ}$ for flexion, abduction, and rotation, respectively, on the normal side and $28^{\circ} \pm 19.8^{\circ}, 31^{\circ} \pm 31.56^{\circ}$, and $48^{\circ} \pm 33.5^{\circ}$ on the involved side $(\mathrm{p}<0.05)$. There was no significant difference between the flexion/extension and rotation movements of the shoulder joint when performing task 1 . However, the difference between flexion and rotation movements of the shoulder joints for the second task was significant ( $>0.05)$.

Conclusions: Those with rotator cuff arthropathy have functional limitations due to muscle weakness and paralysis, especially during the vertical reaching task. However, although these individuals have decreased ROM for transverse reaching tasks, the reduction was not significant.
\end{abstract}

Keywords: Rotator cuff paralysis; Kinematic; Joint movements

\section{INTRODUCTION}

Rotator cuff tear (RCT) arthropathy was first described by Neer [1]. It is classified by subacromial impingement of the humeral head, acetabularization of the acromion, and glenoid erosion after a massive tear of the rotator cuff. This pathology is associated with a decrease in stabilization compressive force across the glenohumeral $(\mathrm{GH})$ joint and superior migration of the humeral head in the direction of the deltoid pull [2-4].

The rotator cuff demonstrates two principal functions of generation of the torque necessary for rotation of the humerus on the glenoid and compression of the humeral head into the gle-

Received: December 30, 2020 Revised: February 25, $2021 \quad$ Accepted: February 27, 2021

Correspondence to: Mohammad Taghi Karimi

Rehabilitation Sciences Research Center, Shiraz University of Medical Sciences, Abiverdi 1, Chamran Avenue, Shiraz 713451733, Iran

Tel: +98-7132625452, Fax: +98-71362564525, E-mail: MT_Karimi@sums.ac.ir, ORCID: https://orcid.org/0000-0001-6162-8131

Financial support: None.

Conflict of interest: None.

CopyrightC 2021 Korean Shoulder and Elbow Society.

This is an Open Access article distributed under the terms of the Creative Commons Attribution Non-Commercial License (http://creativecommons.org/licenses/by-nc/4.0/) which permits unrestricted non-commercial use, distribution, and reproduction in any medium, provided the original work is properly cited. 
noid cavity [5-8]. Although the etiology of rotator cuff arthropathy is unknown, factors such as genetic predisposition, extrinsic impingement and biomechanical imbalance from structures surrounding the cuff, and degeneration changes in tendons have been mentioned in this regard [9]. The incidence of this pathology differs between countries, especially in people older than 50 years. It has been estimated that between $30 \%$ and $70 \%$ of cases of shoulder pain stem from disorders of the rotator cuff. Those with RCT experience superior humeral head translation together with increased superior GH joint force, acromioclavicular joint pressure, and pain when performing daily activities [10,11].

Based on the results of various studies, the kinematics of the shoulder joint are altered significantly in those with RCT, especially during daily activities. GH elevation is reduced in such patients, especially in the context of flexion and abduction. Moreover, they have more scapulothoracic lateral flexion relative to that of normal individuals [1]. In research performed by Kozono et al. [12], full abduction of the shoulder complex in the scapular plane was investigated, and a medial shift at the late phase of motion of the humeral head center was observed. Moreover, during full axial rotation, the humeral head center experienced a greater anterior shift in the patients with rotator cuff arthropathy relative to in the normal matched group [12]. In another study, it was reported that the patients with rotator cuff arthropathy demonstrated an altered and predominantly scapular motion pattern [13]. Most studies have focused on the use of simple motion analysis and reported a decrease in GH joint motion, which might be due to an increase in pain. In other words, patients experience decrease in range of motion (ROM) of the joint as a compensatory mechanism to reduce pain [14-16].

Various approaches have been used to decrease the pain of subjects with RCT and to increase their ROMs, including surgery and conservative treatment (e.g., exercise). Therefore, the main question posed here is the limitations of motions in such a group of patients when performing their daily activities. There seems to be a correlation between RCT and change in kinematics, which might be related to development of symptoms that eventually impact shoulder function. The results of research performed by Parsons et al. [5] showed that GH motion is impaired partially by pain. Those authors observed a significant decrease in scapulohumeral rhythm, a reduction in contribution of scapulothoracic joint motion, and an increase in GH motion. There is no doubt that these patients adopted compensatory mechanisms to improve their performance in daily activities. To our knowledge, no study has evaluated the ROMs of the shoulder joint complex in this group of patients together with the movements produced by muscles when performing simple daily activities. This informa- tion can help clinicians to determine which group or groups of muscles should be strengthened in these patients and also can help surgeons to transfer appropriate muscles to restore the performance of the shoulder complex.

\section{METHODS}

This study was approved by the Institutional Review Board of Shiraz University of Medical Sciences Ethical Committee. Moreover, each subject provided informed consent before data collection.

Five male subjects with massive rotator cuff rupture participated in this study. The men showed a mean age of $62 \pm 5$ years, height of $158 \pm 10 \mathrm{~cm}$, and weight of $58 \pm 8.5 \mathrm{~kg}$. Eligible patients were those with a diagnosis of large to massive RCT. All study participants were scheduled to undergo rotator cuff surgery. The large to massive full thickness of the RCT was confirmed by magnetic resonance imaging prior to study inclusion. Moreover, $\mathrm{X}$-ray imaging was used to classify severity of the disease based on Hameda's classification scheme [12]. According to this classification scheme, all patients exhibited a grade 5 condition (with humeral head collapse). The main exclusion criteria were neuromuscular disorders and previous surgery.

A motion analysis system with eight high-speed cameras was used to record the motions of the shoulder joint complex. Some reflective markers were attached to the anterior superior iliac spine, posterior superior iliac spine, acromioclavicular joint, medial and lateral elbow joints, and medial and lateral styloid processes at the wrist on both the right and left sides. Moreover, markers were attached to the sternum, sternoclavicular joints, C7, and T11. All study participants had RCT on the right side.

The OpenSIM software program (Simbios; Stanford University, Stanford, CA, USA) was used to determine joint ROMs of both the right and left sides. Study participants were asked to complete three tasks, including upward movement of the shoulder joint along the frontal plane with the elbow in an extended position (task 1), upward movement of the shoulder joint in the plane of the scapula with the elbow in $90^{\circ}$ flexion (task 2), and upward movement of the shoulder joint along the sagittal plane (task 3). These motions are considered complex and each one consists of flexion, abduction, and rotation. The tests were performed separately for right and left sides. A well-developed model was used to evaluate the ROM of the shoulder joint in the aforementioned task $[17,18]$. Static test results were collected from the subjects in a sitting position and were used to scale the model in the OpenSIM software program. The Mokka software (Biomechanical ToolKit) program was used to convert the test 
data from C3D to TRC format, which is compatible with the OpenSIM software program. Scaling of the model was completed with an error less than $3 \mathrm{~cm}$. The same procedure was used to collect dynamic test results. Inverse kinematic and inverse dynamic data were used to quantify ROM and joint movements, respectively.

The normal distribution of the parameters was determined by the Shapiro-Wilk test. The difference between ROMs of the joints on the right and left sides and the peaks of the movements of the respective shoulder joints was determined using a two-sample $\mathrm{t}$-test with significance at $\mathrm{p}=0.05$.

\section{RESULTS}

The ROMs of the shoulder joint in task 1 were $93.5^{\circ} \pm 16.5^{\circ}$, $72.1^{\circ} \pm 2.6^{\circ}$, and $103.9^{\circ} \pm 25.7^{\circ}$ for flexion, abduction, and rota- tion, respectively on the normal side, compared with $28^{\circ} \pm 19.8^{\circ}$, $31^{\circ} \pm 31.56^{\circ}$, and $48^{\circ} \pm 33.5^{\circ}$ on the involved side $(\mathrm{p}<0.05)$. There was a significant difference between the normal and involved sides in abduction ROM during task $2(\mathrm{p}=0.025)$. Meanwhile, during task 3, although the ROMs of shoulder flexion/extension, abduction/adduction, and rotation decreased on the involved side relative to on the normal side, the difference was not significant.

Table 1 summarizes the motions of the shoulder joints during these tasks. The mean values of abduction moment of the shoulder joint when performing task 1 were $7.36 \pm 2.7 \mathrm{~N} / \mathrm{m}$ for the normal side and $3.6 \pm 2.2 \mathrm{~N} / \mathrm{m}$ for the involved side, and the difference between these mean values was statistically significant $(\mathrm{p}=0.034)$. There was no significant difference between flexion/ extension and rotation movements of the shoulder joint during task 1 , but this difference was significant $(\mathrm{p}<0.05)$ for the second

Table 1. Mean ROM values of the shoulder joint on the involved (right) and normal (left) sides

\begin{tabular}{|c|c|c|c|}
\hline Variable & Abduction/adduction $\left({ }^{\circ}\right)$ & Flexion/extension $\left(^{\circ}\right)$ & Rotation $\left(^{\circ}\right)$ \\
\hline \multicolumn{4}{|l|}{ Task 1} \\
\hline Normal side & $93.5 \pm 16.5$ & $72.1 \pm 25.6$ & $103.9 \pm 25.7$ \\
\hline RCT side & $28 \pm 19.8$ & $31 \pm 31.56$ & $48 \pm 33.5$ \\
\hline p-value & 0.0 & 0.039 & 0.015 \\
\hline \multicolumn{4}{|l|}{ Task 2} \\
\hline Normal side & $61.5 \pm 19.3$ & $81.4 \pm 20.6$ & $89.4 \pm 44.5$ \\
\hline RCT side & $34.42 \pm 12$ & $64.8 \pm 24.8$ & $53.5 \pm 33.4$ \\
\hline $\mathrm{p}$-value & 0.025 & 0.167 & 0.11 \\
\hline \multicolumn{4}{|l|}{ Task 3} \\
\hline Normal side & $48.8 \pm 41.7$ & $85.2 \pm 49.3$ & $60 \pm 37.9$ \\
\hline RCT side & $15.1 \pm 8.9$ & $32.7 \pm 22.3$ & $25.85 \pm 23.65$ \\
\hline p-value & 0.12 & 0.08 & 0.12 \\
\hline
\end{tabular}

Values are presented as mean \pm standard deviation.

ROM: range of motion, RCT: rotator cuff tear.

Table 2. Mean values of shoulder joint moment on the involved (right) and normal (left) sides

\begin{tabular}{|c|c|c|c|}
\hline Variable & Abduction/adduction (N/m) & Flexion/extension $(\mathrm{N} / \mathrm{m})$ & Rotation $(\mathrm{N} / \mathrm{m})$ \\
\hline \multicolumn{4}{|l|}{ Task 1} \\
\hline Normal side & $7.36 \pm 2.7$ & $2.71 \pm 0.9$ & $1.77 \pm 0.962$ \\
\hline RCT side & $3.6 \pm 2.2$ & $2.85 \pm 1.42$ & $1.77 \pm 1.1$ \\
\hline p-value & 0.034 & 0.4 & 0.0 \\
\hline \multicolumn{4}{|l|}{ Task 2} \\
\hline Normal side & $4.3 \pm 2.77$ & $6.56 \pm 1.59$ & $2.34 \pm 0.77$ \\
\hline RCT side & $2.39 \pm 1.05$ & $4.14 \pm 1.38$ & $1.14 \pm 0.49$ \\
\hline $\mathrm{p}$-value & 0.12 & 0.025 & 0.017 \\
\hline \multicolumn{4}{|l|}{ Task 3} \\
\hline Normal side & $2.35 \pm 1.3$ & $8.1 \pm 1.65$ & $2.3 \pm 0.93$ \\
\hline RCT side & $1.22 \pm 0.37$ & $6.22 \pm 1.62$ & $2.3 \pm 1.32$ \\
\hline p-value & 0.11 & 0.105 & 0.5 \\
\hline
\end{tabular}

Values are presented as mean \pm standard deviation.

RCT: rotator cuff tear. 
task. The mean values of abduction moment of the shoulder joint were $2.35 \pm 1.3 \mathrm{~N} / \mathrm{m}$ on the normal side and $1.22 \pm 0.37 \mathrm{~N} / \mathrm{m}$ on the involved side $(\mathrm{p}=0.11)$. There was no difference between flexion and rotation movements of the shoulder joint when performing task 3 . Table 2 reports the movements of the shoulder joint during the aforementioned tasks.

\section{DISCUSSION}

Patients with rotator cuff arthropathy suffer loss in abilities to perform daily activities with normal ROM. Shoulder motions are mixed and require various degrees of abduction/adduction, flexion/extension, and rotation depending on type of motion. There is not enough evidence in the literature regarding the ROM and movements of the shoulder joint in those with rotator cuff arthropathy. Therefore, this study sought to determine the difference between ROM of the shoulder joint in daily activities on normal and involved sides of the body. The outputs of this research can be used to assess and improve performance of the shoulder complex by way of exercise or surgery.

Our results suggest that those with rotator cuff rupture experience degrees of shoulder joint motion restriction, especially when performing task 1 (abduction) (Table 1). Most individuals can be expected to have problems in completing this task as their ranges of flexion/extension, abduction, adduction, and rotation were decreased significantly. More broadly, our participants had problems performing tasks predominantly requiring motions in the plane above the shoulder joint. For other tasks that can be performed below shoulder level, they experienced less problems (e.g., when performing transverse reaching tasks). However, they also had problems during vertical reaching tasks, as these required greater degree of shoulder abduction and required motions conducted in a plane up to the shoulder joint. The results of kinematic analysis of the shoulder joint support this idea (Table 1). In performing tasks above the shoulder joint, a combination of movements of the GH, scapulothoracic, and other joints in the shoulder joint complex is required. Based on research performed by Zdravkovic et al. [13], those with RCT show irregularities in intra-articular motions, which are essential for motion of the shoulder joint (i.e., patients with RCT showed an altered and predominantly scapular motion pattern).

Movements of the shoulder joint during the mentioned tasks were evaluated in this study, and the participants showed weakness of the abductor muscles when performing an abduction task (vertical reaching, task 1); however, for task 2, they demonstrated weakness of the shoulder flexor. The results of this study support previous studies, confirming that those with rotator cuff injuries demonstrate reduced shoulder flexion and abduction [12,16,19].

Importantly, the decrease in shoulder motion is not simply due to pain, as was mentioned in previous research; it also can be due to weakness in the muscles. This means that those with rotator cuff paralysis cannot complete the ROM of the shoulder joint and have to use some compensatory mechanism such as trunk lean to the contralateral side, which is often not successful for achieving their goal.

Based on the outputs of this study, it can be confirmed that those with rotator cuff injuries have limitations in fulfilling the ROM in the plane above the shoulder joint; however, they show no significant problems with motions below the plane of the scapula. The results of this research highlight that strengthening of the shoulder abductor by physical therapy exercises or tendon transfer (mainly pectoralis, latissimus dorsi) is warranted to restore the abilities of those with such injuries.

There are some limitations that should be acknowledged regarding this study. The main limitation was the small number of participants. Therefore, it is recommended that additional study be performed with a greater number of subjects. Moreover, it is recommended that other types of tasks be evaluated. The results of this study suggest that individuals with RCTs experience degrees of functional limitation due to muscle weakness, especially during vertical reaching tasks. However, although they experience decreased ROM for motions below the plane of the scapula, the difference was not significant. It is recommended that abductor muscles of the shoulder complex be strengthened in this group by way of physical therapy exercises or tendon transfer.

\section{ORCID}

Mohammad Taghi Karimi https://orcid.org/0000-0001-6162-8131

Sahar Khademi https://orcid.org/0000-0002-3330-5789

\section{REFERENCES}

1. Campbell ST, Ecklund KJ, Chu EH, McGarry MH, Gupta R, Lee TQ. The role of pectoralis major and latissimus dorsi muscles in a biomechanical model of massive rotator cuff tear. J Shoulder Elbow Surg 2014;23:1136-42.

2. Guerra-Soriano F, Encalada-Díaz MI, Ruiz-Suárez M, Valero-González FS. Rotator cuff tear athropathy prevalence. Acta Ortop Mex 2017;31:228-32.

3. Feeley BT, Gallo RA, Craig EV. Cuff tear arthropathy: current trends in diagnosis and surgical management. J Shoulder Elbow Surg 2009; 18:484-94.

4. Macaulay AA, Greiwe RM, Bigliani LU. Rotator cuff deficient 
arthritis of the glenohumeral joint. Clin Orthop Surg 2010;2: 196-202.

5. Parsons IM, Apreleva M, Fu FH, Woo SL. The effect of rotator cuff tears on reaction forces at the glenohumeral joint. J Orthop Res 2002;20:439-46.

6. Bassett RW, Browne AO, Morrey BF, An KN. Glenohumeral muscle force and moment mechanics in a position of shoulder instability. J Biomech 1990;23:405-15.

7. Karduna AR, Williams GR, Williams JL, Iannotti JP. Kinematics of the glenohumeral joint: influences of muscle forces, ligamentous constraints, and articular geometry. J Orthop Res 1996;14: 986-93.

8. Saha AK. Dynamic stability of the glenohumeral joint. Acta Orthop Scand 1971;42:491-505.

9. Teunis T, Lubberts B, Reilly BT, Ring D. A systematic review and pooled analysis of the prevalence of rotator cuff disease with increasing age. J Shoulder Elbow Surg 2014;23:1913-21.

10. Geary MB, Elfar JC. Rotator cuff tears in the elderly patients. Geriatr Orthop Surg Rehabil 2015;6:220-4.

11. Sgroi TA, Cilenti M. Rotator cuff repair: post-operative rehabilitation concepts. Curr Rev Musculoskelet Med 2018;11:86-91.

12. Kozono N, Okada T, Takeuchi N, et al. Dynamic kinematics of the glenohumeral joint in shoulders with rotator cuff tears. J Orthop Surg Res 2018;13:9.

13. Zdravkovic V, Alexander N, Wegener R, Spross C, Jost B. How do scapulothoracic kinematics during shoulder elevation differ between adults with and without rotator cuff arthropathy. Clin Orthop Relat Res 2020;478:2640-9.

14. Kwak JM, Ha TH, Sun Y, Kholinne E, Koh KH, Jeon IH. Motion quality in rotator cuff tear using an inertial measurement unit: new parameters for dynamic motion assessment. J Shoulder Elbow Surg 2020;29:593-9.

15. Kijima T, Matsuki K, Ochiai N, et al. In vivo 3-dimensional analysis of scapular and glenohumeral kinematics: comparison of symptomatic or asymptomatic shoulders with rotator cuff tears and healthy shoulders. J Shoulder Elbow Surg 2015;24: 1817-26.

16. Kolk A, Henseler JF, de Witte PB, et al. The effect of a rotator cuff tear and its size on three-dimensional shoulder motion. Clin Biomech (Bristol, Avon) 2017;45:43-51.

17. Wu W, Lee PV, Bryant AL, Galea M, Ackland DC. Subject-specific musculoskeletal modeling in the evaluation of shoulder muscle and joint function. J Biomech 2016;49:3626-34.

18. Seth A, Dong M, Matias R, Delp S. Muscle contributions to upper-extremity movement and work from a musculoskeletal model of the human shoulder. Front Neurorobot 2019;13:90.

19. Mell AG, LaScalza S, Guffey P, et al. Effect of rotator cuff pathology on shoulder rhythm. J Shoulder Elbow Surg 2005;14(1 Suppl S):58S-64S. 